\title{
Multi-year maintenance planning framework using multi-attribute utility theory and genetic algorithms
}

\author{
Zaharah Allah Bukhsh* ${ }^{*}$, Irina Stipanovic and Andre G. Doree
}

\begin{abstract}
This paper introduces a comprehensive framework for the development of optimal multi-year maintenance plans for a large number of bridges. A maintenance plan is said to be optimal when, within the given budget, a maximum number of bridges can be maintained in the best possible year, achieving maximum performance with minimum socio-economic impact. The framework incorporates heuristic rules, multi-attribute utility theory, discrete Markov chain process, and genetic algorithms to find an optimal balance between limited budgets and performance requirements. The applicability of the proposed framework is illustrated on an extensive case study of highway bridges. The framework enables asset owners to execute various planning scenarios under different budget and performance requirements, where each resulting plan is optimal. The focus of this study has mainly been on highway bridges, however the framework is general and can be applied to any other infrastructure asset type.
\end{abstract}

Keywords: Maintenance planning, Multi-objectives, Optimization, Genetic algorithms, Markov decision processes, Multi-attribute, Utility function, Bridges

\section{Introduction}

Bridges are one of the most fundamental structures on a road transport network. They provide crossings at critical locations, reduce travel times, and maintain traffic flow. Since most of the road infrastructure in Europe was built after the 1950s, many road bridges are reaching their critical age, while being exposed to adverse climate effects and increased public demands. Because of limited financial resources, agencies have to take careful investment and maintenance planning decisions to improve safety, serviceability, and availability of infrastructure while minimizing overall life-cycle costs.

Within the theme of maintenance planning and optimization, literature studies can be divided into three groups; reliability assessment by deterioration modeling [1], comparisons of different maintenance alternatives [2-5] and multi-objective optimization techniques [6-8]. Most of these approaches are focused on individual bridge level, while few consider maintenance

*Correspondence: z.allahbukhsh@utwente.nl

Department of construction management and engineering, Universiteit

Twente, Enschede, Netherlands planning at the network level. Though these advance optimization approaches are promising, they are computationally expensive, often non-transparent and can be challenging to implement by asset owners. Many agencies use a Bridge Management Systems (BMS) to develop maintenance plans by allocating the budget, which is still mainly driven by subjective ranking and preferences of domain experts $[9,10]$. These systems typically employ single-objective optimization analysis to allocate budget, but do not take into account other performance aspects [11].

To develop accurate yet simple maintenance planning solutions constituting of multiple performance goals and resource constraints, search-based optimization techniques like Genetic Algorithms (GA) are popular. A brief overview of the notable studies that employ GA for maintenance planning is considered herein. Bocchini and Frangopol [12] developed a probabilistic framework to schedule preventive maintenance for bridges with a focus on reliability assessment. A two-stage maintenance planning method for a large number of bridges is proposed in [13]. First, a preventive maintenance plan of a bridge is optimized, and then, the total life-cycle cost is reduced 
by allocating flexible intervention intervals. Denysiuk et al. [14] proposed a computational framework consisting of degradation and maintenance models to search for optimal maintenance schedules. Similarly, Xie et al. [15] developed a framework to find the optimal initial and consecutive time intervals between maintenance activities in order to minimize the life-cycle environmental impact.

The literature provides numerous maintenance planning approaches varying by the employed degradation models and optimization techniques. Some of the past studies have also been the source of motivation for this study $[2,16,17]$. However, it is noted that the searchbased optimization solution considers the multiple objectives of maintenance planning but neglect to scrutinize the subjective preferences of the asset owners/decisionmakers [18]. Moreover, most of the planning solutions are illustrated on the small set of assets, where, with the best of our knowledge, none of the maintenance planning approaches provides a comprehensive methodology to plan the maintenance of hundreds of network assets in an end-to-end manner. This study aims to mitigate these gaps by introducing a Multi-year Maintenance Planning Framework (MMPF). The purpose of the MMPF is to find the best time for the maintenance of bridges by developing an optimal schedule over a multi-year planning period while accommodating decision makers' preferences and satisfying multiple performance goals.

The key contributions of this study are as follows:

- Employing the multi-attribute utility theory to incorporate multiple-objectives and decision-makers' preferences for the ranking of a large number of bridges.

- Applying Markov chain process in conjunction with genetic algorithms for the performance forecasting of bridges for each year.

- Illustrating the applicability of the proposed framework on a case study of highway bridges.

- Introducing a comprehensive two-step multiobjective optimization for the maintenance planning based on current condition state only and the future predicted condition states.

- Enabling asset owners to execute various maintenance planning scenarios under varying budget and performance constraints

The paper is structured as follows: Section 2 introduces the proposed MMPF along with details of techniques and algorithms applied. Section 3 and Section 4 illustrate the application of the MMPF on case study data, outline the implementation details, and analyze the numerical results. The discussion of results, potential limitations of MMPF, and concluding remarks of the study are provided in Section 5 .

\section{Methodology of Multi-year Maintenance Planning Framework}

This section introduces the methodology of the Multiyear Maintenance Planning Framework (MMPF), which seeks to develop optimal maintenance plans for a number of infrastructure assets.

Figure 1 presents a flowchart of MMPF to highlight the interaction of employed techniques and algorithms. The proposed MMPF mainly constitutes of four modules; i) an impact assessment module to decide on the type of maintenance intervention and its resulting impact ii) a Multi-Attribute Utility Theory (MAUT) module for ranking of bridges based on preference uncertainty and risk attitude of a decision-maker, iii) a Markov Chain Process (MCP) based performance prediction module to forecast the condition of a bridge in the future and finally iv) a Genetic Algorithm (GA) module to develop numerous multi-year maintenance plans in order to find the optimal solution.

\subsection{Problem formulation}

The proposed framework considers multiple objectives of maintenance planning. The principal objectives are to maximize the performance level of bridges and to minimize the maintenance cost by optimally planning the maintenance treatments. The objectives and constraints of the maintenance planning problem are represented as:

$$
\begin{aligned}
& \text { minimise } \sum_{t=1}^{T} \sum_{b=1}^{B} I\left(x_{t, b}\right) \\
& \text { minimise } \sum_{t=1}^{T} \sum_{b=1}^{B} c\left(x_{t, b}^{m}\right) \\
& \text { subject to } \sum_{t=1}^{T} \sum_{b=1}^{B} I\left(x_{t, b}\right) \leq \text { Threshold } \\
& \text { subject to } \sum_{t=1}^{T} \sum_{b=1}^{B} c\left(x_{t, b}^{m}\right) \leq \text { Budget }
\end{aligned}
$$

where $I\left(x_{t, b}\right)= \begin{cases}I\left(x_{t, b}\right)-z, & \text { if } x_{b t}=m . \\ I\left(x_{t, b}\right)+f(d), & \text { otherwise, apply MCP. }\end{cases}$

The aforementioned parameters used for modeling are defined as follows:

$$
\begin{aligned}
B & =\text { Number of bridges } \\
T & =\text { Number of years in planning horizon } \\
I\left(x_{t, b}\right) & =\text { Condition index of a bridge } b \text { in year } t
\end{aligned}
$$




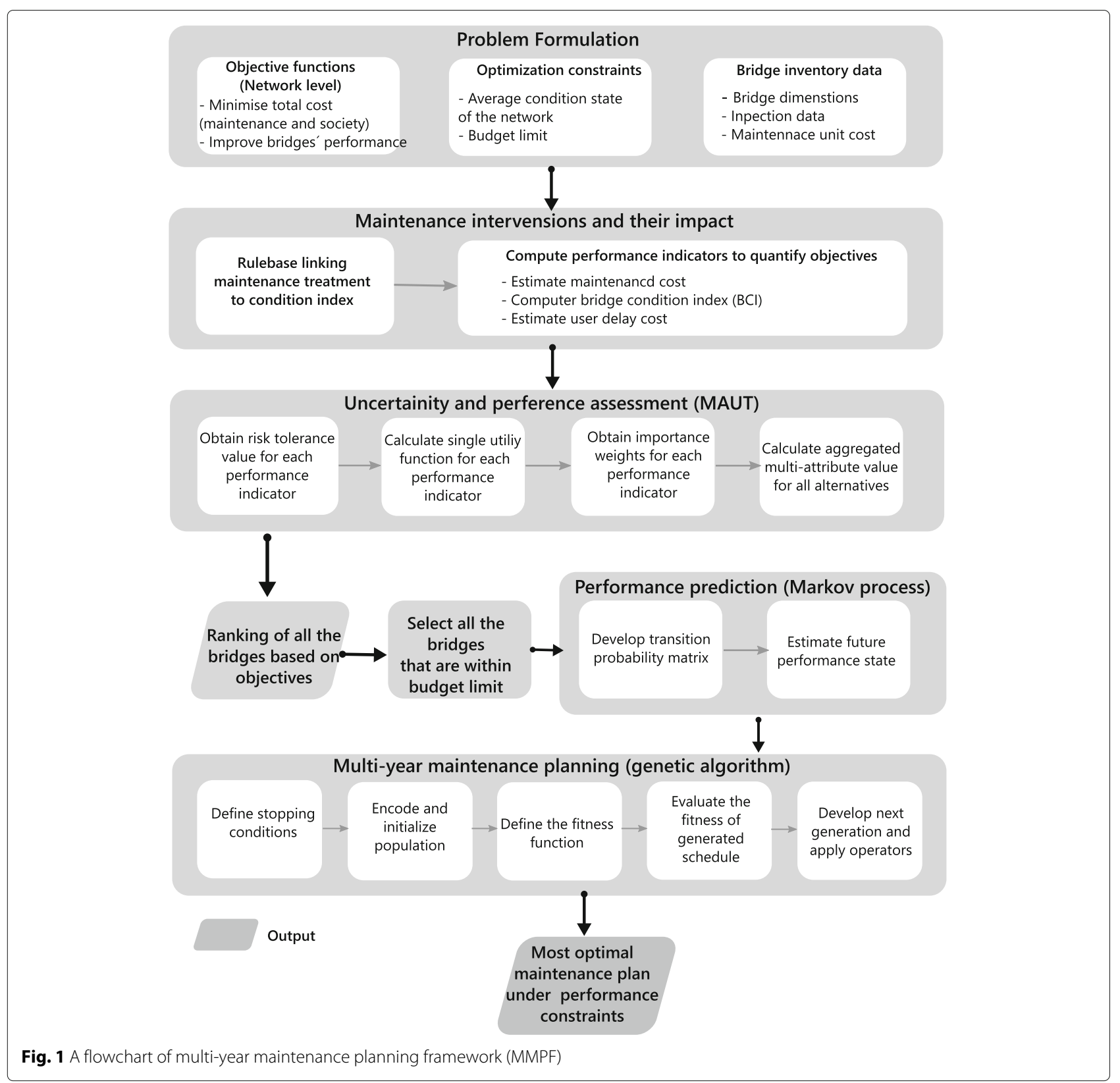

$c\left(x_{t, b}^{m}\right)=$ Cost function to compute cost of maintenance activity $m$ performed on a bridge $b$ in year $t$

Threshold $=$ Condition index threshold of the bridges of network $z=$ Improved condition state of a bridge after maintenance/remediation measure (based on Table 1)

$f(d)=$ Function based on MCP to forecast the condition of a bridge
With this formulation, Eq. (1a) represents the first objective function, which aims to minimize the condition score $I$ of all the bridges $B$ over the finite planning period $T$. The minimization function is applied because, in a discrete condition scorecard, 1 represents the best (as new) condition state, whereas 6 represents the worst condition (loss of function). If assigned with a maintenance activity in a year, the state of the bridge will be improved (represented as $z$ ); otherwise, it is deteriorated with a certain degree $f(d)$ complying to Markov chain transition matrices represented in Eq. (1e). Equation (1b) represents the second objective function, which is targeted for 
the minimization of maintenance cost resulted from $m$ maintenance activity. Both objectives must be reached within the two main constraints. Firstly, all the bridges must be below the specified condition index threshold value as represented in Eq. (1c). This condition enforces the allocation of maintenance activity to bridges having poor condition states, irrespective of their high rehabilitation cost. Secondly, the accumulated costs of planned maintenance activities on the selected bridges must be within the specified budget limit denoted by Eq. (1d). Currently, the impact of maintenance activities on the users is not considered as an objective with the fixed value. Instead, it is used as the criteria to minimize the impact on the users by determining the user delay costs resulting from maintenance activities, while accounting for bridge importance in terms of the number of vehicles per day, the extended travel times, and duration of different maintenance options.

\subsection{Maintenance intervention and quantification of its impact}

This section introduces heuristics to link condition states with various maintenance treatments and provides a procedure to quantify the socio-economic impacts of maintenance activity.

\subsubsection{Condition state and maintenance treatments}

Bridges are deteriorating and experiencing various damages due to the environmental impact, aging and traffic loading. The observed damages and their severity define the condition state of a bridge and consequently the required maintenance treatments. Table 1 provides a simplified rule-based choice of maintenance treatments related to the Bridge Condition Index (BCI). We consulted following studies [2, Section 3.5], [20, Table 1], [21, Table 1 and 2] and [19, D03] to define the respective maintenance activities, their impact, and associated condition ranges.

\subsubsection{Performance indicators quantification}

This section provides a procedure to quantify the impact of maintenance activity on different aspects (reliability, availability, and economy), by quantifying performance indicators as follows:

- Element-level to system-level BCI: BCI represents the overall health of the structure. Since bridges have multiple elements with distinct damage types, many road agencies inspect the bridges at an element level [22]. However, the maintenance decisions must be made at the system-level (as a whole bridge); therefore, the element-level condition indices must be translated to represent the overall health of a structure. We applied the weighted-average method [22] in which an expert establishes the importance of each element to another. The choices of an expert are based on the
Table 1 Heuristics defining maintenance treatments based on condition state ranges [19, D03]

\begin{tabular}{llll}
\hline $\begin{array}{l}\text { Condition } \\
\text { range }(\mathrm{BCl})^{*}\end{array}$ & $\begin{array}{l}\text { Treatment } \\
\text { name }\end{array}$ & Treatment details & Impact on $\mathrm{BCl}$ \\
\hline $1-2$ & Nothing & No action needed & No change \\
$2-2.7$ & Monitoring & Monitoring and inspection & No change \\
& $\begin{array}{l}\text { Minor } \\
\text { intervention }\end{array}$ & &
\end{tabular}

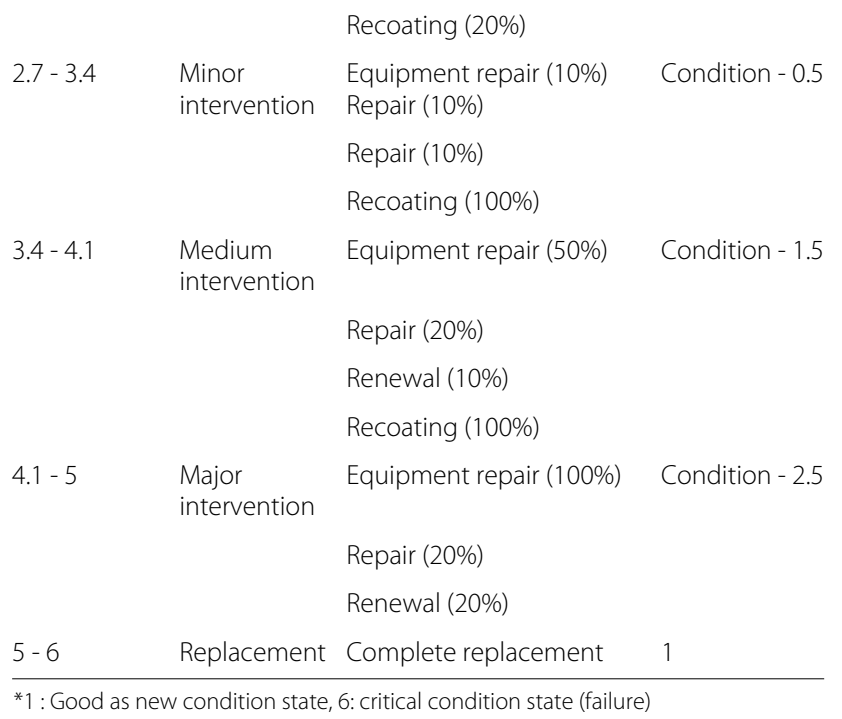

elements' failure history, maintenance frequency, and the impacts on the bridge in case of failure. Table 2 provides the elicited relative importance of the bridge elements. Using the relative importance values, $\mathrm{BCI}$ is calculated by the following Equation:

$$
B C I=\sum_{i=1}^{n}\left(C I_{i} * W_{i}\right)
$$

where $n$ represents number of bridge elements, $C I_{i}$ is condition index of element $i$ obtained through experts judgements after (visual) inspection, and $W_{i}$ is weighted importance of element $i$ elicited by an expert as shown in Table 2.

Table 2 Weighted score of bridge elements [23]

\begin{tabular}{lll}
\hline No. & Elements & Weighted score \\
\hline 1 & Superstructure & 0.3185 \\
2 & Bearings & 0.2104 \\
3 & Abutment & 0.1813 \\
4 & Joints & 0.1288 \\
5 & Pavement & 0.0618 \\
6 & Railing & 0.0510 \\
7 & Guardrail & 0.0478 \\
\hline
\end{tabular}


- Maintenance cost: The maintenance cost is a monetary value borne by an agency as a result of performing maintenance activities. The maintenance cost of each bridge is computed as a sum of the unit costs of all the maintenance treatments $\left(U C A_{m}\right)$ multiplied by the quantity $\left(Q_{m}\right)$ or the volume of the treated area. The formula to calculate the maintenance cost is provided as follows:

$$
M C=\sum_{m=1}^{n} U C A_{m} *\left(Q_{m}\right)
$$

where $M C$ is total maintenance cost spend on a bridge and $n$ is number of bridge's element.

- User delay cost: The user delay cost estimates the impact of a maintenance treatment on the availability of the bridge and highlights its importance on the network. It represents the value of extended travel time of the road users due to work-zones in a monetary form. The number of factors contributes to user delay cost [24], expressed as follows:

$$
\begin{aligned}
U D C & =E T T * A H T_{h} * V_{o h} * D_{h} \\
E T T & =\frac{L}{V_{r}}-\frac{L}{V_{s}}
\end{aligned}
$$

where ETT is extra travel time due to speed restrictions, $A H T_{h}$ is average traffic per hour passing over the bridge on a working day, $V_{o h}$ is value of an hour of the user time, $D_{h}$ is duration of the maintenance activity, $L$ is length of working zones $(\mathrm{km}), V_{s}$ is standard velocity $(\mathrm{km} / \mathrm{h})$ and $V_{r}$ is reduced velocity due to maintenance.

\subsection{Uncertainty and preference assessment using MAUT}

This module incorporates the preference uncertainty and risk attitude of experts, for maintenance planning, by employing the principles of Multi-Attribute Utility Theory (MAUT). The concept of the utility function is inspired by gambling, wherewith equal probability to obtain the best value or worst value, a gambler/decisionmaker needs to take certain risks [25]. The purpose of MAUT in MMPF is threefold: 1) to accommodate multiple performance indicators, ranging from societal to economic aspects, 2) to enable decision-makers to state their maintenance preferences under uncertainty and 3) to assign a rank to each bridge by performing a trade-off of multiple performance objectives. The resulting prioritization filters-out the broad set of bridges from the road network that do not fulfill the objectives, and therefore, must not be scheduled for maintenance in the near future. The ranking can also be utilized for next year's maintenance plan as the highest-ranked bridges present the urgent need of maintenance prompted by either low cost or poor condition state.

In the following, a brief explanation and application steps of the MAUT process is provided
1. This study applies the Exponential Utility Function (EUF) to elicit the utility scores of each attribute since it captures the preference uncertainty and Risk Tolerance (RT) of a decision maker [26]. The formula to calculate the utility scores $U_{a}$ of each attribute a for the alternatives $x$ is provided below:

$$
U_{a}\left(x_{a}\right)=A-B * e^{\left(\frac{-x_{a}}{R T}\right)}
$$

where $A$ and $B$ are scaling constants, and $e$ is an exponential constant. The EUF requires the decisionmakers to respond to the lottery question of a maximum and a minimum value of an attribute, where the indifference point has to be reached between the best and the worst possible outcome. Such an indifference point for a decision-maker is referred to as the Certainty Equivalent (CE).

2. The risk tolerance is calculated based on expected value $(E V)$ and $C E$, where $E V$ is median of worst and best value of attribute values. The value $C E$ is chosen by the experts based on following the principle:

Risk Attitude $=\left\{\begin{array}{l}\text { Risk Neutral, if } E V=C E \text { (Linear shaped) } \\ \text { Risk Avoiding, if } E V>=C E \text { (Concave shaped }) \\ \text { Risk Taking, if } E V<C E \text { (Convax shaped })\end{array}\right.$

3. Trade-offs are performed among attributes a by assigning the relative importance $k$ based on the preferences of the decision-maker.

$$
k_{a}=\frac{\operatorname{rate}\left(x_{a}\right)}{\sum_{a=1}^{A}\left(x_{a}\right)}
$$

where $\operatorname{rate}\left(x_{a}\right)$ is a weight assigned by a decision maker to attribute a, $\sum_{a=1}^{A}\left(x_{a}\right)$ is sum of all the weights given to attributes and $k$ represents attribute's relative importance.

4. The utility score of each attribute and their assigned weight factors are aggregated to elicit the final ranking of the alternatives by computing additive aggregation as follows:

$$
U(x)=\sum_{i=1}^{n} k_{a} U_{a}\left(x_{a}\right)
$$

where $k_{a}$ is the relative importance of attribute, $U_{a}\left(x_{a}\right)$ is single attribute utility for each attribute a for an alternative $x$.

The computed MAUT score establishes the ranking of each alternative. Based on the minimization objective function, the alternatives with lower aggregated MAUT scores will obtain a higher ranking.

\subsection{Performance prediction using Markov chain process}

It is essential to forecast the performance of assets in order to optimally plan the maintenance activities and to estimate the cost. Based on mathematical and statistical 
principles, multiple deterministic and stochastic models have been proposed for deterioration modelling [1, 27]. For this study, the discrete Markov Chain Process (MCP) is applied to model the random and uncertain process of bridge deterioration statistically, as already illustrated by several studies [28-30].

Markov chain consists of set of states denoted as $S=s_{1}, s_{2}, . ., s_{n}$, where each state represents the bridge condition state. The process starts from one state $s$ and moves to another state $s^{\prime}$, with a certain probability of $p$. The probabilities $p$ are called transition probabilities, which quantify the probability of a bridge or its element to move from one state $s$ to another state $s^{\prime}$. There are multiple procedures to compute the transition probability matrices, namely expected-value method [31], binomial regression [32], and ordered probit model [33]. The simplest technique, requiring minimum data, is the percentage prediction method, which forecasts the percentage of total bridges belonging to each condition state [34]. The calculation procedure can be represented as:

$$
p\left(s, s^{\prime}\right)=\frac{\sum_{n=1}^{N}\left(s, s^{\prime}\right)}{\sum_{n=1}^{N}(s)}
$$

where $n_{s s^{\prime}}$ is number of elements transitioning from condition state $s$ to state $s^{\prime}$ and $n_{s}$ is total number of elements having state $s$.

The computed transition probabilities are expressed in a matrix of size $w^{*} w$, where $w$ is the number of discrete condition state. The performance prediction of the bridges using the MCP is used to decide on a specific year for the maintenance execution.

\subsection{Multi-year maintenance planning using genetic algorithms}

The development of the maintenance plan is a multiobjective combinatorial optimization problem, with the objectives to reduce the life cycle costs of assets and fulfill the performance requirements over a specified planning horizon. Instead of traditional optimization techniques, which are complicated and time-consuming, we choose to apply a Genetic Algorithm (GA) solution that is efficient and provides robust results. GA is a combinatorial optimization search technique motivated by Darwinian evolution theory of natural selection, genetics and survival-of-the-fittest [35].

In GA, the potential solutions are expressed as individuals, which consist of combinations of chromosomes. The chromosomes are finite-length strings, which represent the decision variable of a search problem. Unlike traditional optimization approaches, GA generates a population of potential solutions iteratively until the predetermined population size is reached. The objective function must evaluate each individual of the population in order to distinguish good solutions from bad solutions. Further stochastic operators i.e., cross-over, the mutation is applied on selected populations based on their relative fitness score assigned by objective function to generate next population. Figure 2 provides a flow chart of GA.

Depending on the problem, different encoding schemes such as binary, tree-based, value, and permutation can be selected [36]. The optimization problem of this study is encoded in the value scheme since the fitness of a generated schedule depends only on the discrete values, i.e., cost and condition irrespective of a specific order. Each of the generated solutions is evaluated for its fitness by the objectives function, established in problem formulation Section 2.1.

\section{Case Study}

The proposed MMPF is validated on a case study of concrete bridges, which are part of a highway network ${ }^{1}$. The database contains data about location, bridge structure, materials, element-level condition scores resulting from principle inspections, records of performed and planned maintenance treatment(s) and their unit costs. The agency uses condition data, damage estimation, and expert judgments implemented into risk assessment to decide on future maintenance plans. Considering that the existing procedure still heavily relies on subjective judgments, it is not fully transparent or easy to follow. The proposed MMPF primarily utilizes existing condition score data and can be applied within several agencies.

A five-year maintenance plan is programmed with the aim to keep the network-level bridge score of at least 2.7 with the estimated budget of $€ 6$ million only. The objective is to improve the performance of the bridges by minimizing the socio-economic impact under a limited budget. We have considered five treatment options, namely i) monitoring, ii) minor intervention, iii) medium intervention, iv) major intervention, and v) complete replacement. The available treatment options are linked to the range of BCI, as shown in Table 1 .

For this case, seven essential elements of a bridge are considered (see Table 2). Since the principal inspection is performed at the element-level every six years, each element has two visually assessed indices from the data of the past 12 years. To have a thorough understanding of bridge structural integrity and its deterioration over time, first, the transition probabilities are calculated. Next, the case study data is used to compute the performance indicators.

\subsection{Computing transition probability matrix}

The transition probabilities of each element are calculated by percentage prediction method, provided in Section 2 .

\footnotetext{
${ }^{1}$ The case study data is provided by a road agency with a non-disclosure agreement.
} 


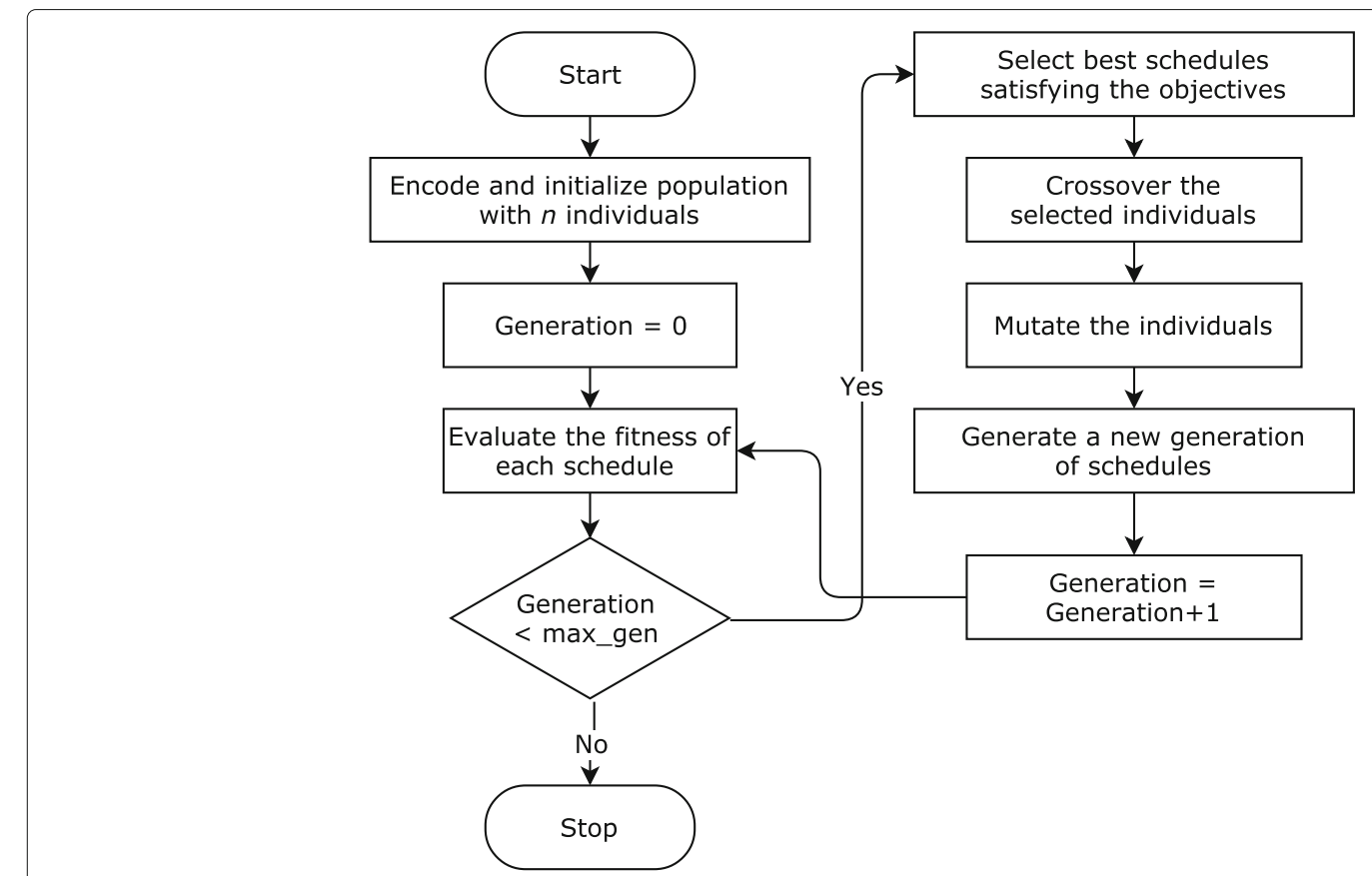

Fig. 2 Flowchart of a typical genetic algorithm

The percentage prediction method mainly relies on the number of changes in condition state between the inspection interval. We have considered only those elements' data that show the deteriorating trend in condition state, which means no maintenance has been performed between the two inspection activities. The transition probability is represented as $p\left(s, s^{\prime}\right)$, where $s \leq s^{\prime}$.

By using Eq. 8, the transition probability matrix for each bridge component is computed. Next, the system-level probability matrix is computed using the relative importance of each bridge element (provided in Table 2). The system-level transition probability matrix is provided in Table 3, where the leftmost column represents the current condition, and the top row shows the future condition states.

Most of the elements had condition state 4 as their lowest (poor) condition, except for joints and railing. To

Table 3 System level transition probability matrix of bridges

\begin{tabular}{lllllll}
\hline & 1 & 2 & 3 & 4 & 5 & 6 \\
1 & 0.0315 & 0.795 & 0.090 & 0.075 & 0.004 & 0 \\
2 & 0 & 0.832 & 0.081 & 0.072 & 0.0144 & 0 \\
3 & 0 & 0 & 0.779 & 0.188 & 0.031 & 0 \\
4 & 0 & 0 & 0 & $\mathbf{0 . 7 0}$ & $\mathbf{0 . 2 5}$ & $\mathbf{0 . 0 5}$ \\
5 & 0 & 0 & 0 & 0 & 0.179 & $\mathbf{0 . 8 2 1}$ \\
6 & 0 & 0 & 0 & 0 & 0 & $\mathbf{1}$ \\
\hline
\end{tabular}

The bold values represent the transition probabilities elicited from historical data and literature, and other probabilities are obtained from the case study data. eliminate this skewed data distribution, we have used historical data about bridge performance from other bridge management system to estimate probabilities of transitions from condition 4 to 5 and 5 to 6 [19]. The elicited probability scores are denoted with bold text in Table 3 .

\subsection{Computing performance indicators}

Performance indicators quantify the objective of optimal multi-year maintenance planning. These indicators measure the impact of maintenance activity concerning the maximization of bridge network performance (covering structural aspects), and minimization of socio-economic costs. By utilizing the quantification procedure provided in Section 2.2.2, we have calculated the BCI, maintenance cost, and user delay cost for all bridges of the case study.

Figure 3 presents the range of $\mathrm{BCI}$ found in the case study dataset. The $\mathrm{BCI}$ of most of the bridges ranges between 1.5 to 3.5 , except for few bridges having greater than 3.5 condition score. This limited dispersion of $\mathrm{BCI}$ in the dataset shows the overall good condition state of the considered bridges.

The approximate cost of maintenance treatment of each bridge is computed using the Eq. 3 and the rules defined in Table 1. Figure 4 shows a boxplot of the maintenance cost with respect to the condition state. The bridges having a poor condition state require significant maintenance and higher cost as compared to bridges having relatively better condition state. In other words, a bridge is economical to maintain in its initial state of damage compared to the critical damage level. 


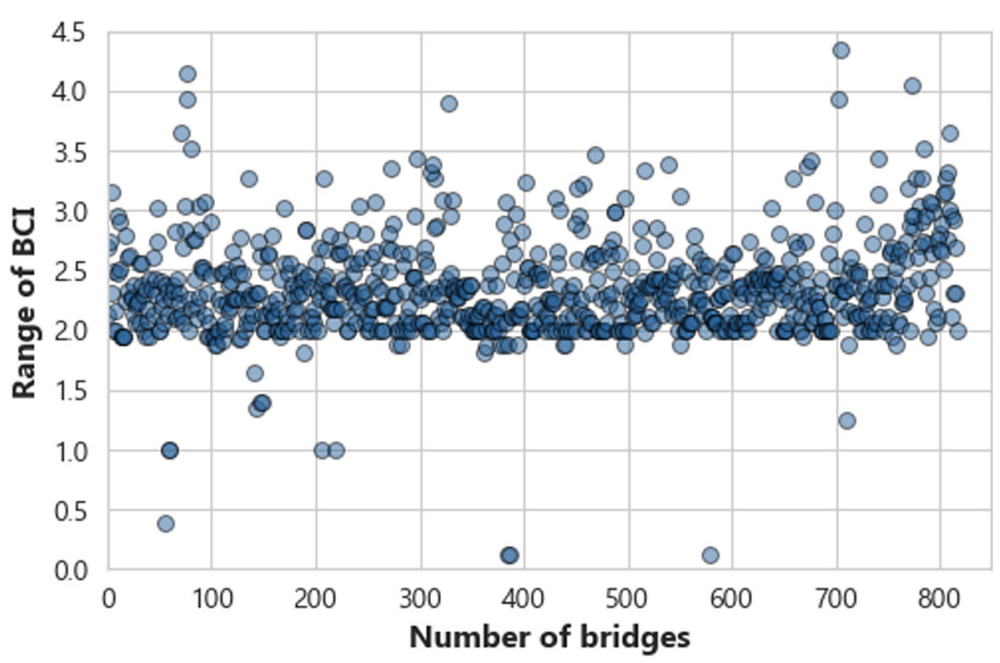

Fig. 3 Ranges of $\mathrm{BCl}$

The user delay cost is calculated by using the Eq. 4, where the length of the work zone is estimated to be three times the total length of a bridge. The reduced speed due to maintenance is $90 \mathrm{~km} / \mathrm{h}$ whereas the standard speed is $130 \mathrm{~km} / \mathrm{h}$. The duration of various maintenance interventions are estimated as $48 \mathrm{~h}$ for minor, $168 \mathrm{~h}$ for medium, and $312 \mathrm{~h}$ for the major treatments. Finally, the average traffic per hour over a bridge is extracted by considering the bridge location. The range of user delay cost of bridges with respect to their bridge's length and duration of the maintenance activity is provided in Fig. 5.

\section{Framework Implementation and Numerical Results}

As a result of computing performance indicators, the bridges having $\mathrm{BCI}$ lower than 2.7 are eliminated from the analysis, meaning they are in good condition and do not need maintenance in the next five-years. In the following sections, the implementation details of MAUT, MCP, and genetic algorithms applied to the case study data are provided.

\subsection{Implementation of Multi-attribute utility theory}

MAUT method is applied to the remaining 123 bridges. The first step of MAUT is to determine the single utility function (SUF) of each attribute across all the alternatives (bridges), as discussed in Section 2.3. The calculation of SUF for each attribute is an extensive process. Therefore, for the sake of brevity, the computation process is omitted here. An interested reader may refer to [23] for the detailed procedure.

Next, the weight for each of the attributes is defined to describe their impact on the overall objective. The highest importance is assigned to the $\mathrm{BCI}$ with 90 , followed by

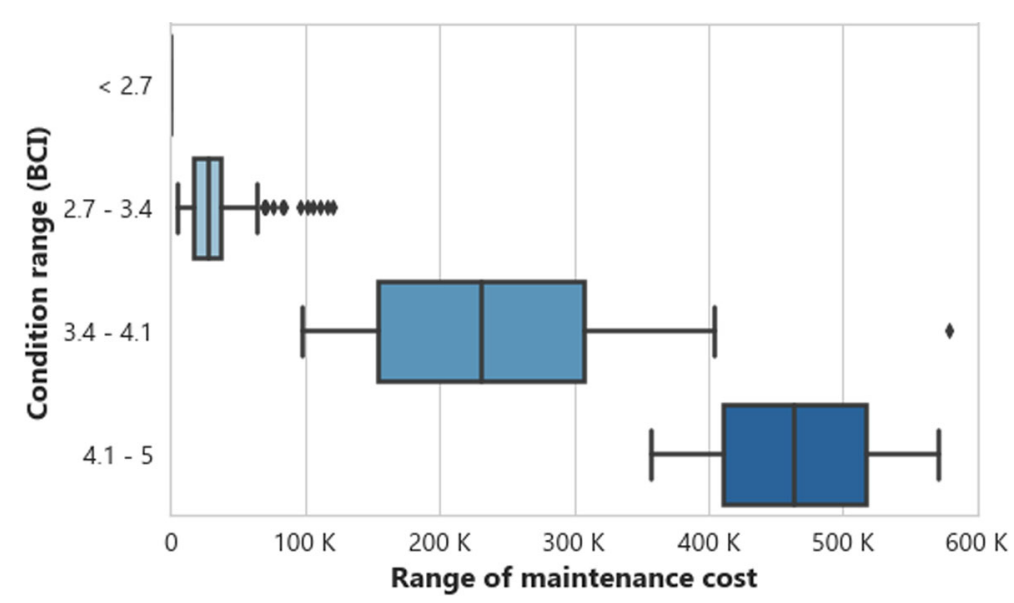

Fig. 4 Range of computed maintenance cost 


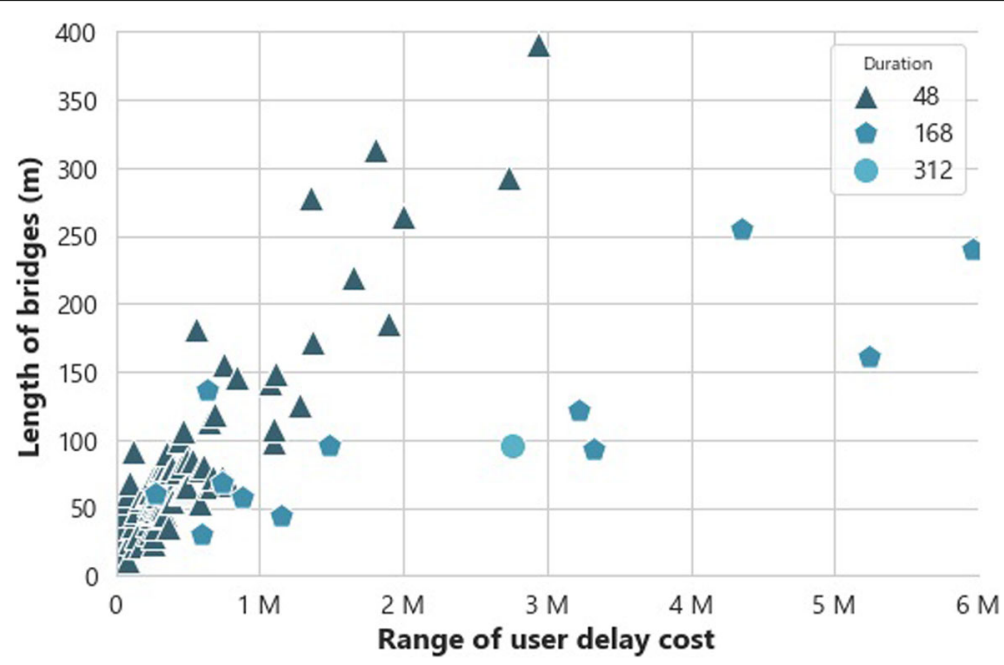

Fig. 5 Range of computed user delay cost: The duration depicts the number of hours required for different maintenance interventions

the maintenance cost with 70 , and the UDC with 60 value. The relative and normalized importance weights are then calculated by using Eq. 6 . From the utility function of attributes, a global aggregated score is computed for each alternative by Eq. 7. The global aggregated score ranks all the 123 bridges in an order where multiple objectives are taken into account, as previously explained.

To ensure readability, Fig. 6 provides the MAUT scores and ranking of the top 40 bridges. Bridge $(B 80)$ is 77 years old and has a deck area of approximately $2000 \mathrm{~m}^{2}$, $\mathrm{BCI}$ of 4.13 , and the highest user delay cost is ranked as number one requiring a major intervention. The lowestranked bridges (not included in Fig. 6) are mostly below 30 years of age, have small deck areas, low user delay costs due to their lower traffic intensities, and require only minor interventions. Considering the budget constraint of $€ 6$ million, we have performed a cumulative sum of the maintenance costs of the ranked bridges and selected only

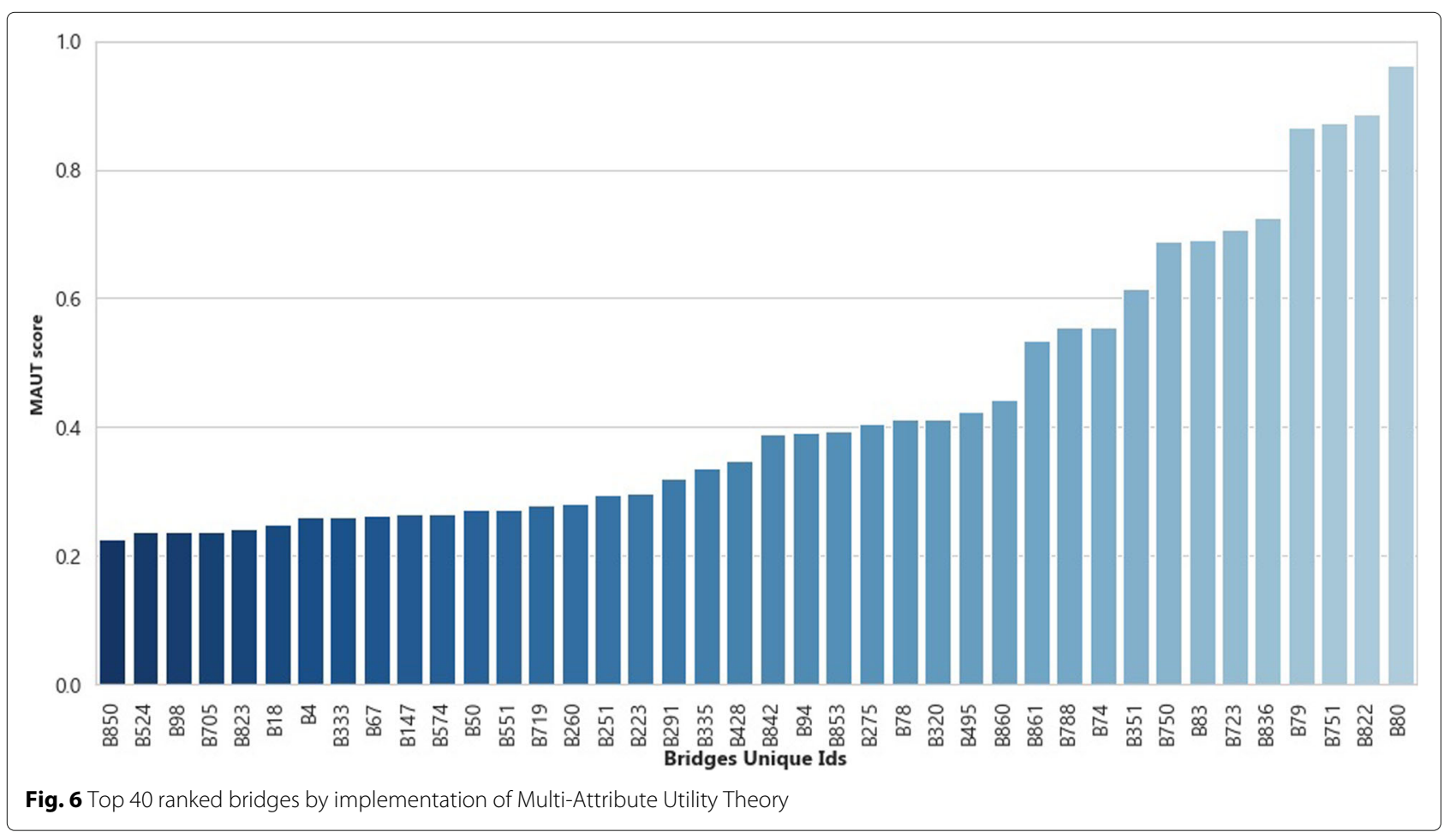


those bridges that can be maintained within the available budget over 5 years, which resulted in the selection of 28 bridges in total.

\subsection{Implementation of genetic algorithms and Markov chain process}

The Genetic Algorithm (GA) is applied to the 28 selected bridges to seek an optimal maintenance plan for the next five years. To apply GA on the maintenance planning problem, we have utilized the evolutionary computation framework DEAP, available for Python programming language [37]. A population size of 150 individuals with ten generations is adopted for the GA simulator. A partially matched crossover method with $0.2 \%$ probability is used, where the chromosomes of two individuals are randomly swapped to generate two new and unique individuals (i.e., maintenance plans). Similarly, a mutation probability of $0.7 \%$ is applied in which, instead of mutating the chromosomes values, the order of the chromosomes is shuffled.

Moreover, for the selection of the best individuals among the number of generated individuals, a non-dominated multi-objective optimization algorithm (NSGA-II) is applied [38]. The GA simulator is tuned multiple times with varying cross-over and mutation probabilities to find the settings that best converge for the given problem.

Figure 7 shows the number of individuals (maintenance plans) generated for a single population on the axis of condition state and budget limit having varying fitness levels. A maintenance plan which compromises on the required performance threshold of 2.7 can have as low budget as $€ 2.1$ million while comparing to those plans which achieve 2.14 of performance level but on the cost of $€ 6.6$ million. The grey (dotted) lines in Figure 7 show the optimization constraints for the 5 year maintenance plan, where condition scores of all the bridges on the network should be at least 2.7 , while taking account the $€ 6$ million budget limit. All the maintenance plans depicted with the 'blue plus' marker are feasible solutions that fulfill the defined constraints. Though it is worthwhile to mention that few generated maintenance plans do not allocate all the 28 bridges for planning, therefore present lower cost. The solutions represented with 'red circle' are infeasible solutions and do not comply with the defined optimization constraints.

Among the 150 maintenance plans (individuals) generated in 10 iterations (generations), we choose the single most optimal maintenance plan as presented in Table 4 . The plan exhibits the set of bridges allocated to a specific year along with treatment name, maintenance cost, and improved condition state. The treatment name refers to the intervention details provided in Table 1 . As mentioned earlier, the bridge which is not maintained in a particular year (e.g., 2020) will decline in condition state until it is selected for maintenance. For instance, $B 861$ had a condition value of 3.65 at the time of maintenance planning (say 2018), while when scheduled to be maintained in year 5 (say 2022), it is estimated to have deteriorated condition score by 4.16. By the optimal allocation of bridge maintenance to specific years, the plan shows a budget of $€ 5.9$ million and achieving the average condition score for all bridges of 2.27.

For the sake of comparison and to establish a baseline, we also generated sequential maintenance plan without applying the GAs. The purpose of sequential plan is to mimic usual planning scenario where a bridge with poor condition state is maintained first. An assumption of equal budget allocation to each year is made, where the residual budget of any year is equally distributed in all

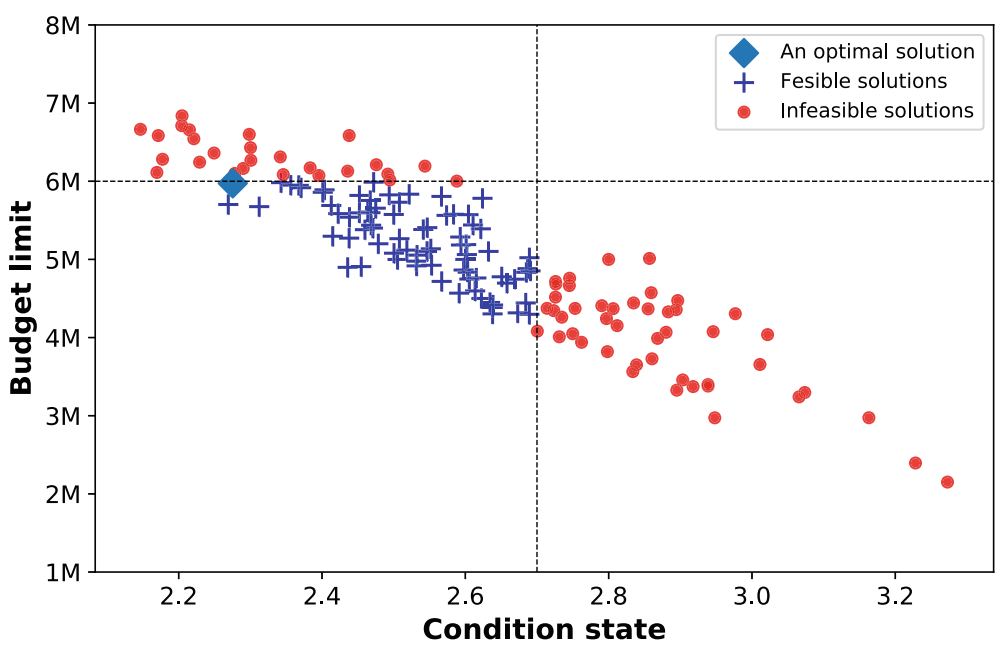

Fig. 7 All the maintenance plans generated by GAs 
Table 4 Most optimal maintenance plan of 28 bridges using MMPF

\begin{tabular}{|c|c|c|c|c|c|}
\hline No & Bridge name & Current condition & Treatment name & Maintenance cost & Improved condition \\
\hline \multicolumn{6}{|c|}{ Year 1} \\
\hline 1 & B74 & 3.65 & Medium intervention & $147,336.58$ & 2.15 \\
\hline 2 & B719 & 3.36 & Minor intervention & $30,257.6$ & 2.61 \\
\hline 3 & B291 & 3.35 & Minor intervention & $49,702.03$ & 2.6 \\
\hline 4 & B822 & 4.04 & Medium intervention & $578,944.5$ & 2.54 \\
\hline 5 & B223 & 3.27 & Minor intervention & $63,608.32$ & 2.52 \\
\hline 6 & B842 & 3.07 & Minor intervention & $95,551.68$ & 2.32 \\
\hline 7 & B723 & 3.42 & Medium intervention & $404,047.16$ & 1.92 \\
\hline \multirow[t]{3}{*}{8} & B428 & 3.24 & Minor intervention & $76,259.75$ & 2.49 \\
\hline & Yearly summary & \multicolumn{4}{|c|}{ Total yearly spending: 1,445,707.62 (24\%) } \\
\hline & & \multicolumn{4}{|c|}{ Remaining budget: 4,554,292.38 (76\%) } \\
\hline \multicolumn{6}{|c|}{ Year 2} \\
\hline 9 & B275 & 3.12 & Minor intervention & $102,202.4$ & 2.37 \\
\hline 10 & B853 & 2.93 & Minor intervention & $110,745.5$ & 2.18 \\
\hline 11 & B335 & 3.47 & Medium intervention & $242,006.54$ & 1.97 \\
\hline 12 & B79 & 4.08 & Medium intervention & $356,609.97$ & 2.58 \\
\hline 13 & B78 & 3.08 & Minor intervention & $120,244.48$ & 2.33 \\
\hline 14 & B750 & 4.08 & Medium intervention & $222,261.69$ & 2.58 \\
\hline \multirow[t]{3}{*}{15} & B351 & 4.05 & Medium intervention & $175,173.64$ & 2.55 \\
\hline & Yearly summary & \multicolumn{4}{|c|}{ Total cost spent: 1,329,244.21 (22\%) } \\
\hline & & \multicolumn{4}{|c|}{ Remaining budget: 3,225,048.17 (53\%) } \\
\hline \multicolumn{6}{|c|}{ Year 3} \\
\hline 16 & B94 & 2.93 & Minor intervention & $116,274.03$ & 2.18 \\
\hline 17 & B495 & 3.68 & Medium intervention & $119,176.0$ & 2.18 \\
\hline 18 & B836 & 3.75 & Medium intervention & $238,610.66$ & 2.25 \\
\hline 19 & B260 & 3.15 & Minor intervention & $55,593.66$ & 2.4 \\
\hline \multirow[t]{3}{*}{20} & B80 & 4.14 & Major intervention & $570,867.62$ & 1.64 \\
\hline & Yearly summary & \multicolumn{4}{|c|}{ Total cost spent: 1,100,521.97 (18\%) } \\
\hline & & \multicolumn{4}{|c|}{ Remaining budget: 2,124,526.20 (35\%) } \\
\hline \multicolumn{6}{|c|}{ Year 4} \\
\hline 21 & B751 & 4.35 & Major intervention & $356,589.91$ & 1.85 \\
\hline 22 & B251 & 2.98 & Minor intervention & $82,777.45$ & 2.23 \\
\hline \multirow[t]{3}{*}{23} & B83 & 3.88 & Medium intervention & & 2.38 \\
\hline & Yearly summary & \multicolumn{4}{|c|}{ Total cost spent: 696,271.83 (11\%) } \\
\hline & & \multicolumn{4}{|c|}{ Remaining budget: 1,428,254.37 (23\%) } \\
\hline \multicolumn{6}{|c|}{ Year 5} \\
\hline 24 & B861 & 4.16 & Major intervention & $254,416.0$ & 1.66 \\
\hline 25 & B860 & 3.71 & Medium intervention & $582,257.7$ & 2.21 \\
\hline 26 & B551 & 3.74 & Medium intervention & $175,820.0$ & 2.24 \\
\hline 27 & B788 & 3.89 & Medium intervention & $291,239.04$ & 2.39 \\
\hline \multirow[t]{5}{*}{28} & B320 & 3.89 & Medium intervention & $97,676.26$ & 2.39 \\
\hline & Yearly summary & \multicolumn{4}{|c|}{ Total cost spent: 1,401,409 (23\%) } \\
\hline & & \multicolumn{4}{|c|}{ Remaining budget: 26,845 (0.044\%) } \\
\hline & 5-Years summary & \multicolumn{4}{|c|}{ Total cost spent: 5973154.6 (99\%), Remaining budget: 26845(0.044\%) } \\
\hline & & \multicolumn{4}{|c|}{ Number of maintained bridges: 28, Avg. condition: 2.27} \\
\hline
\end{tabular}

the remaining years of planning. This sequential maintenance planning, based on condition states only, can allocate only 18 bridges for maintenance with average performance level of 2.58 within the given budget limit of $€ 6$ million. Bridges based on their condition score were sorted in a descending order and all the bridges which are within the yearly budget limit are allocated for maintenance. 


\section{Discussion and Conclusions}

This paper introduces a comprehensive framework for the development of optimal maintenance planning for a network of road bridges over a multi-year planning period, while considering the multiple objectives of performance requirements and budget constraints. The proposed Multi-year Maintenance Planning Framework (MMPF) employs multi-attribute utility theory for ranking a large number of bridges by capturing the preference uncertainty and risk attitude of a decision-maker. It develops several maintenance plans by using genetic algorithms in conjunction with Markov chain processes. The MMPF is generic and can include any other performance attributes, according to the owners' needs (e.g., safety, environment, maintainability, etc).

In addition to developing an optimal maintenance plan, the proposed framework enables asset owners to execute various maintenance planning scenarios by changing budget limits and performance objectives for the network. Additionally, the framework can be used for future budget planning by forecasting condition states of bridges. The framework is validated using an inventory of concrete bridges on a highway network to develop an optimal 5-year maintenance plan given a budget constraint and a condition threshold. Based on the given criteria, out of $800+$ bridges, a group of 123 bridges was found to have condition states below the required performance level. The application of the framework prioritizes the bridges and generate an optimal maintenance plan for 28 bridges within the given budget limit, whereas, the sequential maintenance plan (i.e., a bridge with the poor condition is always maintained first), applied to the same set of bridges, enables the maintenance of 18 bridges only.

The proof-of-concept on the case study data expresses the usefulness of MMPF; however, there are few limitations related to the scope and used methods. For the detailed overview on the impact on users in case of (maintenance) interventions, the MMPF must be extended with traffic flow modeling. The proposed framework develops the static maintenance plan by filtering the bridges that require maintenance. Here the assumption is that filtered-out bridges are in good condition state and will not require maintenance in the next five years. However, factors like extensive usage, environmental impacts may escalate deterioration process of assets, thus resulting in an unexpected need for maintenance.

Regarding the methodology, the transition probability matrix developed in this study demands data of at least the last two inspections and expects the ideal distribution of data over time. However, in reality, not all the bridge elements can have detailed inspection records for all the classes, which makes the calculation of transition probability matrices a difficult activity. Similarly, despite easy implementation and reasonable running times for the genetic algorithm, it is well-known that they are unable to guarantee an optimal solution due to possibly ample solution space of a combinatorial multi-objective problem. However, genetic algorithms still promise good quality solutions in a reasonable time, given the complexity of multiple objectives and constraints.

From the application perspective, tool support is needed to enable the seamless execution of various maintenance planning scenarios. To encourage tool development and to support the reproducibility, the implementation code python has been made publicly available ${ }^{2}$. The future work of this study aims to improve the MMPF further by considering the specific structural aspects of each bridge while defining specific maintenance treatment. Another potential improvement of MMPF is to employ the models of machine learning for the performance prediction of each bridge on the bases of particular bridge characteristics, condition and maintenance history, and usage intensity. This will also enable a more accurate estimation of the required budget for the owners.

\section{Authors' contributions}

ZAB introduced the framework, analyzed the case study data and was a major contributor in writing the manuscript. IS guided the framework design process and reviewed the results as they developed. AD reviewed the overall manuscript. All authors read and approved the final manuscript.

\section{Funding}

This study has been performed under funding from the European Union's Horizon 2020 research and innovation program with grant agreement No. 636285 DESTination Rail.

\section{Availability of data and materials}

The datasets generated and/or analysed in this study are not publicly available due non-disclosure agreement with the road agency. But the sample dataset and the Python implementation code of the framework will be made available on GitHub repository after the peer-review process.

\section{Competing interests}

The authors declare that they have no competing interests.

Received: 12 July 2019 Accepted: 3 December 2019

Published online: 09 January 2020

\section{References}

1. Alaswad, S., \& Xiang, Y. (2017). A review on condition-based maintenance optimization models for stochastically deteriorating system. Reliability Engineering \& System Safety, 157, 54-63.

2. Ghodoosi, F., Abu-Samra, S., Zeynalian, M., Zayed, T. (2017). Maintenance cost optimization for bridge structures using system reliability analysis and genetic algorithms. Journal of Construction Engineering and Management, 144(2), 04017116.

3. Hu, X., \& Madanat, S. (2014). Determination of optimal mrr policies for retaining life-cycle connectivity of bridge networks. Journal of Infrastructure Systems, 21(2), 04014042.

4. Kong, J.S., \& Frangopol, D.M. (2003). Life-cycle reliability-based maintenance cost optimization of deteriorating structures with emphasis on bridges. Journal of Structural Engineering, 129(6), 818-828.

5. Liu, M., \& Frangopol, D.M. (2004). Optimal bridge maintenance planning based on probabilistic performance prediction. Engineering Structures, 26(7), 991-1002.

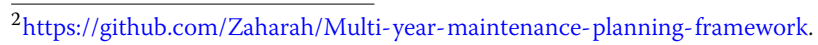


6. Barone, G., \& Frangopol, D.M. (2013). Hazard-based optimum lifetime inspection and repair planning for deteriorating structures. Journal of Structural Engineering, 139(12), 04013017.

7. Dong, Y., \& Frangopol, D.M. (2016). Probabilistic time-dependent multihazard life-cycle assessment and resilience of bridges considering climate change. Journal of Performance of Constructed Facilities, 30(5), 04016034.

8. Kim, S., \& Frangopol, D.M. (2017). Efficient multi-objective optimisation of probabilistic service life management. Structure and Infrastructure Engineering, 13(1), 147-159.

9. Markow, M.J., \& Hyman, W.A. (2009). Bridge Management Systems for Transportation Agency Decision Making Vol. 397: National Academies Press. https://doi.org/10.17226/14270.

10. Mirzaei, Z., Adey, B.T., Klatter, L., Kong, J.S. (2012). Overview of existing Bridge Management Systems-Report by the IABMAS Bridge Management Committee, In 6th International Conference on Bridge Maintenance, Safety and Management (IABMAS 2012): International Association for Bridge Maintenance And Safety (IABMAS)

11. Bush, S., Henning, T.F., Ingham, J.M., Raith, A. (2014). An agent-based framework for improved strategic bridge asset management, In Proceedings OfThe 9Th Austroads Bridge Conference (Abc 2014): Bridges Of The Future: Sydney Olympic Park, New South Wales, 22-25 October 2014.

12. Bocchini, P., \& Frangopol, D.M. (2011). A probabilistic computational framework for bridge network optimal maintenance scheduling. Reliability Engineering \& System Safety, 96(2), 332-349.

13. Furuta, H., Nakatsu, K., Ishibashi, K., Miyoshi, N. (2014). Optimal bridge maintenance of large number of bridges using robust genetic algorithm, In Structures Congress 2014. https://doi.org/10.1061/9780784413357.200: American Society of Civil Engineers.

14. Denysiuk, R., Fernandes, J., Matos, J.C., Neves, L.C., Berardinelli, U. (2016). A computational framework for infrastructure asset maintenance scheduling. Structural Engineering International, 26(2), 94-102.

15. Xie, H.B., Wu, W.-J., Wang, Y.-F. (2018). Life-time reliability based optimisation of bridge maintenance strategy considering Ica and Icc. Journal of Cleaner Production, 176, 36-45.

16. Chootinan, P., Chen, A., Horrocks, M.R., Bolling, D. (2006). A multi-year pavement maintenance program using a stochastic simulation-based genetic algorithm approach. Transportation Research Part A: Policy and Practice, 40(9), 725-743.

17. Frangopol, D.M., Dong, Y., Sabatino, S. (2017). Bridge life-cycle performance and cost: analysis, prediction, optimisation and decision-making. Structure and Infrastructure Engineering, 13(10), 1239-1257.

18. Almeida, d.A.T., Ferreira, R.J.P., Cavalcante, C.A.V. (2015). A review of the use of multicriteria and multi-objective models in maintenance and reliability. IMA Journal of Management Mathematics, 26(3), 249-271. https://doi.org/10.1093/imaman/dpv010.

19. Alten, K., Deix, S., Peelen, W., Wessels, J., Courage, W., Ravnikar Turk, M., Skaric Palic, S., Acalin, N., Lundkvist, S.-O. (2012). Asset service condition assessment methodology (ASCAM Project), In 4th European pavement and asset management conference-EPAM. Linköping: Swedish National Road and Transport Research Institute.

20. Morcous, G., \& Lounis, Z. (2005). Maintenance optimization of infrastructure networks using genetic algorithms. Automation in Construction, 14(1), 129-142.

21. Lee, C.-K., \& Kim Sung, K. (2007). Ga-based algorithm for selecting optimal repair and rehabilitation methods for reinforced concrete (rc) bridge decks. Automation in construction, 16(2), 153-164.

22. Chase, S.B., Adu-Gyamfi, Y., Aktan, A., Minaie, E., et al. (2016). Synthesis of national and international methodologies used for bridge health indices. Technical report, Federal Highway Administration.

23. Allah Bukhsh, Z., Stipanovic, I., Klanker, G., O'Connor, A., Doree, A.G. (2018) Network level bridges maintenance planning using multi-attribute utility theory. Structure and infrastructure engineering, 1-14.

24. Wang, Y., \& Goodrum, P.M. (2005). Use of conceptual road user costs for a rapid roadway construction decision making system, In Construction Research Congress 2005: Broadening Perspectives (pp. 1-11).

25. Keeney, R.L., \& Raiffa, H. (1993). Decisions with Multiple Objectives: Preferences and Value Tradeoffs. Cambridge, UK: Cambridge University Press.
26. Keeney, R., \& von Winterfeldt, D. (2007). M13 practical value models. In R.F.W. Miles (Ed.), Advances in Decision Analysis: From Foundations to Applications. von Winterfeldt, D. (pp. 232-252). New York: Cambridge University Press.

27. Ahmad, R., \& Kamaruddin, S. (2012). A review of condition-based maintenance decision-making. European Journal of Industrial Engineering, 6(5), 519-541. https://doi.org/10.1504/EJIE.2012.048854. PMID: 48854.

28. Cesare, M.A., Santamarina, C., Turkstra, C., Vanmarcke, E.H. (1992). Modeling bridge deterioration with markov chains. Journal of Transportation Engineering, 118(6), 820-833.

29. Grinstead, C.M., \& Snell, J.L. (2012). Introduction to Probability: American Mathematical Society: Providence, Rhode Island, United States.

30. Robelin, C.-A., \& Madanat, S.M. (2007). History-dependent bridge deck maintenance and replacement optimization with markov decision processes. Journal of Infrastructure Systems, 13(3), 195-201.

31. Jiang, Y., Saito, M., Sinha, K.C. (1988). Bridge performance prediction model using the markov chain. Transport Research Record, 1(1180), 25-32.

32. Madanat, S., \& Ibrahim, W.H.W. (1995). Poisson regression models of infrastructure transition probabilities. Journal of Transportation Engineering, 121(3), 267-272.

33. Madanat, S., Mishalani, R., Ibrahim, W.H.W. (1995). Estimation of infrastructure transition probabilities from condition rating data. Journal of infrastructure systems, 1(2), 120-125.

34. Setunge, S., \& Hasan, S. (2011). Concrete bridge deterioration prediction using markov chain approach, In In Proceedings of the International Conference on Structural Engineering, Construction and Management (ICSECM), Kandy, Sri Lanka, 15-17 December 2011 (pp. 1-14).

35. Holland, J.H. (1975). Adaptation in natural and artificial systems. an introductory analysis with application to biology, control, and artificial intelligence. Ann Arbor, MI: University of Michigan Press, 439-444.

36. Kumar, A. (2013). Encoding schemes in genetic algorithm. International Journal of Advanced Research in IT and Engineering, 2(3), 1-7.

37. Fortin, F.-A., Rainville, F.-M.D., Gardner, M.-A., Parizeau, M., Gagne, C. (2012). Deap: Evolutionary algorithms made easy. Journal of Machine Learning Research, 13, 2171-2175.

38. Deb, K., Agrawal, S., Pratap, A., Meyarivan, T. (2000). A fast elitist non-dominated sorting genetic algorithm for multi-objective optimization: Nsga-ii, In Parallel Problem Solving from Nature PPSN VI. https://doi.org/10.1007/3-540-45356-3_83 (pp. 849-858): Springer.

\section{Publisher's Note}

Springer Nature remains neutral with regard to jurisdictional claims in published maps and institutional affiliations.

\section{Submit your manuscript to a SpringerOpen ${ }^{\circ}$ journal and benefit from:}

- Convenient online submission

- Rigorous peer review

- Open access: articles freely available online

- High visibility within the field

- Retaining the copyright to your article

Submit your next manuscript at $>$ springeropen.com 